\title{
FLOW BEHAVIOR ANALYSIS OF CONFINED FLUID IN A FLEXIBLE TUBE: APPLICATION OF ASYMPTOTIC APPROACH
}

\author{
A. MEHDARI ${ }^{1}$, M. AGOUZOUL ${ }^{2}$, M. HASNAOUI ${ }^{3}$ \\ 1. Mohammed V University, ERG2 (ME), Rabat, Morocco - abdmehdari@gmail.com \\ 2. Mohammed V University, ERG2 (ME), Rabat, Morocco - agouzoul@emi.ac.ma \\ 3. Royal Air Force School, SMD, Marrakech, Morocco - hasnaouimohammed@ hotmail.com
}

\begin{abstract}
In this paper, we asymptotically investigate a confined fluid flow in a flexible tube with a variable section. The fluid is considered to be newtonian, incompressible and it elapses in elastic and isotropic shell. This study provides a review of recent analysing the effects of the elastic wall tube properties over the fluid behaviour. The unsteady fluid flow will be analysed following the singular perturbations theory according to a large Reynolds number and a small aspect radio. The wall is assumed to be a thin shell that generate a small axisymmetric vibration. This model is mathematically developed by using the thin shell linear theory that is governed by a geodesic curvature parameter.
\end{abstract}

Keywords: flow Behaviour, confined, elastic tube, asymptotic approach.

\section{Introduction}

The fluid flow through an elastic tube is important in many areas of industry. This configuration is observed in several fields: aeronautical sector [1], biology [2], the renewable energies [3], and recently vibratory analysis of flexible riser under the effects of oil dynamic behaviour [4].

It should be noted that numerical methods often fail where asymptotic approaches are perfectly successful, especially so-called "stiffs" problems. Asymptotic analysis consists of constructing approximate mathematical models, basing on hierarchical construction of asymptotic constraints with the aim to obtain a substantial solution.

In this purpose, the asymptotic analysis of the unsteady flow is paired with a small dimensionless parameter characterizing the aspect ratio of the tube. It governs the asymptotic expansion based on singular perturbations approach. Moreover, the dynamic equilibrium of the tube is developed by the linear approach of the thin shell theory founded on geodesic curvature parameter.

\section{Problem Formulation}

\subsection{Fluid}

We analyze an unsteady flow of an incompressible, viscous and newtonian fluid in an symmetric axial cylindrical domain. $\rho$ and $v$ denote respectively the density and the kinematic viscosity of fluid, $\mathrm{L}$ is the tube length and $R_{0}$ is the radius at rest. $R\left(z^{\prime}, t^{\prime}\right)$ is the dynamic radius (i.e. radius is a function of the longitudinal variable and time).

We assume that the tube behaves as a homogenous, isotropic, linear and elastic thin shell with thickness $h$ (Figure 1) and $\rho_{T}$ is the tube density (Figure 1).

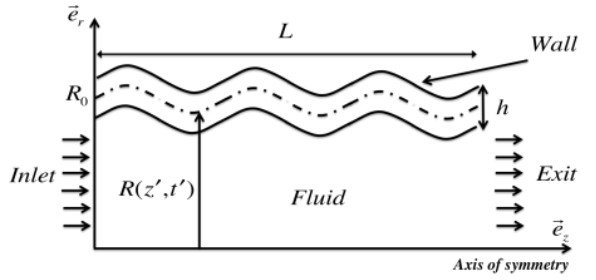

Figure 1. Deformed domain

The physical variables are denoted with the quote sign. At this level, we introduce dimensionless variables, namely:

$\left\{r=\frac{r^{\prime}}{R_{o}} ; \mathrm{z}=\frac{z^{\prime}}{L} ; \mathrm{t}=\frac{t^{\prime}}{T_{f}} ; u=\frac{u^{\prime}}{W_{o}} ; w=\frac{w^{\prime}}{W_{o}} ; p=\frac{p^{\prime}}{{ }^{2} W_{o}^{2}}\right.$

$T_{f}$ is the reference time, $\quad\left(=R_{0} / L\right) \square 1$ is the aspect ratio and $W_{o}$ represents the reference axial velocity.

Using system (1), the dimensionless Navier-Stokes and continuity equations of the problem read as:

$$
\left\{\begin{array}{l}
\left(\frac{S_{t}}{-}\right) \frac{\partial u}{\partial t}+\left(u \frac{\partial u}{\partial r}+w \frac{\partial u}{\partial z}\right)=\frac{\partial p}{\partial r}+\left(\frac{R_{e}{ }^{1}}{2}\right)\left\{\frac{1}{r} \frac{\partial}{\partial r}\left(r \frac{\partial u}{\partial r}\right)+{ }^{2} \frac{\partial^{2} u}{\partial z^{2}} \frac{u}{r^{2}}\right\} \\
\left(\frac{S_{t}}{-}\right) \frac{\partial w}{\partial t}+\left(u \frac{\partial w}{\partial r}+w \frac{\partial w}{\partial z}\right)={ }^{2} \frac{\partial p}{\partial z}+\left(\frac{R_{e}{ }^{1}}{\partial}\right)\left\{\frac{1}{r} \frac{\partial}{\partial r}\left(r \frac{\partial w}{\partial r}\right)+{ }^{2} \frac{\partial^{2} w}{\partial z^{2}}\right\} \\
\frac{\partial u}{\partial r}+\frac{\partial w}{\partial z}+\frac{u}{r}=0
\end{array}\right.
$$

The numbers figuring in (2) are the Reynolds number $R_{e}\left(=R_{o} W_{o} /\right)$ and the Strouhal number $S_{t}\left(=R_{o} /\left(W_{o} T_{f}\right)\right)$.

At large Reynolds number and low Strouhal number, the singular perturbations analysis of the system (2) reveals the following asymptotic constraints:

$S_{t} O\left(\right.$ ) et $R_{e}{ }^{1} O()$

\subsection{Tube}


According to the Kirchhoff-Love assumptions [5], the flexible tube is modelled by using the thin shell linear theory [6]. The following system formulates the dimensionless variables and parameters:

$$
\left\{\begin{array}{c}
{ }_{0}=\frac{h}{R_{0}} ; \quad \bar{R}=\frac{R}{R_{0}} ; \quad t=\frac{t^{\prime}}{T_{T}} ; \quad{ }_{1}=\frac{h}{L} ; \quad{ }_{F}=\frac{W_{0}^{2}}{{ }_{1}{ }_{2}} \\
\bar{P}_{3}^{*}=\frac{P_{\text {int }} P_{e x t}}{. W_{0}^{2}{ }^{2}} ; \quad-\quad=\frac{\bar{u}_{0.2}}{L} ; \quad{ }_{T}=\frac{{ }_{T} \cdot L^{2}}{\left({ }_{1}+{ }_{2}\right) \cdot T_{T}^{2}} ; \quad H=\frac{2}{{ }_{1}+{ }_{2}}
\end{array}\right.
$$

Where $T_{T} \simeq T_{f}$ is the tube time reference, ${ }_{1}$ and ${ }_{2}$ are the Lame constants and $\bar{u}_{0.2}$ is the wall axial displacement reference.

Dimensionless analysis of the equilibrium equations, based on the Least Degeneracy Principle, states to following asymptotic constraints.

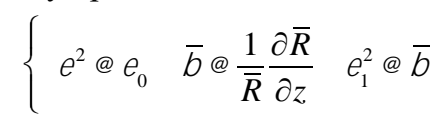

Where $\frac{1}{\bar{R}} \frac{\bar{R}}{z}$ is the tube geodesic curvature along the $\vec{e}_{z}$. According the system (4), the governing equations for the tube motion are expressed as the under system:

$$
\left\{\begin{array}{l}
{ }_{H} H \frac{\partial \bar{u}_{2}^{*}}{\partial z}{ }^{-}{ }^{2} \bar{u}_{2}^{*}+{ }_{T}{ }_{1 / 3} \bar{R} \frac{\partial^{2} \bar{R}}{\partial t^{2}}\left(1 \frac{1}{\bar{R}}\right) \quad{ }_{F} \bar{R} \bar{P}_{3}^{*}=0 \\
\frac{\partial^{2} \bar{u}_{2}^{*}}{\partial z^{2}}+{ }^{-} \bar{u}_{2}^{*}+{ }_{T}{ }^{-1 / 3} \cdot \bar{R} \frac{\partial^{2} \bar{R}}{\partial t^{2}} \quad{ }^{-7 / 3} \bar{R}^{2} \quad(H+1)^{-7 / 3} \bar{R}^{2} \\
+{ }^{7 / 3} \bar{R}^{2}+\left(1 \frac{1}{\bar{R}}\right)=0
\end{array}\right.
$$

$\bar{u}_{2}^{*}$ is the dimensionless wall axial displacement.

\section{Linearization and resolution of the problem}

Fluid: Let us linearize equations (2) about the particular solution at the inlet of tube. The Least Degeneracy Principle provides us the following form:

$$
u=\varepsilon^{3} u^{*}(r, z) e^{I \omega t} ; w=1+\varepsilon^{3} w^{*}(r, z) e^{I \omega t} ; p=\tilde{P}_{a m b}+\varepsilon^{3} p^{*}(r, z) e^{I \omega t}
$$

Where $u^{*}, w^{*}, p^{*}$ are, respectively, the perturbed radial and axial velocities, and pressure:

$$
u^{*}=w^{*}=p^{*}=0 \text { for } \quad r=z=0
$$

Using (7), the solution is given by the following system:

$$
\left\{\begin{array}{c}
u^{*}=A \cdot J_{1}\left(\frac{1}{2} \sqrt{2} \sqrt{I} \cdot r\right) \cdot z+F_{2}(r) \\
w^{*}=\frac{1}{4} \sqrt{2} \sqrt{I} \cdot A \cdot J_{0}\left(\frac{\sqrt{2} \sqrt{I}}{2} \cdot r\right) z^{2}+z\left[A_{1} J_{0}(\sqrt{I} \cdot r)\right] \\
p^{*}=\frac{1}{2} \sqrt{2} \sqrt{I}\left[A \cdot J_{0}\left(\frac{1}{2} \sqrt{2} \sqrt{I} \cdot r\right)\right] z+F_{4}(r) \\
F_{2}(r)=\frac{I . A \cdot J_{1}\left(\frac{1}{2} \sqrt{2} \sqrt{I} r\right)}{+A_{2} J_{1}(\sqrt{I} r)} \\
F_{4}(r)=A_{1} J_{0}(\sqrt{I} r)+\frac{I \cdot A_{2} J_{0}(\sqrt{I} r)+\sqrt{2} A J_{0}\left(\frac{\sqrt{2}}{2} \sqrt{I} r\right)}{\sqrt{I}}+A_{3}
\end{array}\right.
$$

Where the $J_{0}$ and $J_{1}$ are the Bessel functions and $\omega$ is the fluid frequency. $A, A_{1}, A_{2}$ and $A_{3}$ are the complex numbers with $\mathrm{I}$ is the imaginary unit:

$$
|A| \quad 1 \text { and }] 0,30]
$$

Tube: We take up the linearization process around the initial equilibrium state $\left({ }^{-} \square 1\right)$ :

$\bar{P}_{3}^{*}={ }^{-1 / 3} \bar{P}_{3}^{0} ; \quad \bar{R}=1+{ }^{-1 / 3} \bar{R}_{1}, \quad \bar{u}_{2}^{*}=\bar{u}_{2.0}^{*}+{ }^{-} \bar{u}_{2.1}^{*}$

$\bar{P}_{3}^{0}, \bar{u}_{2.0}, \bar{u}_{2.1}$ and $\bar{R}_{1}$ are the perturbed parameters. Approached solution is formulated at the $0^{\text {th }}$ order of ${ }^{-1 / 3}$ by:

$\left\{\begin{array}{c}\left.\gamma_{F} p^{*}\right|_{r=1}+\bar{R}_{1}=0 \\ \frac{\partial^{2} \bar{u}_{2.0}^{*}}{\partial z^{2}}-\frac{\gamma_{T}}{2} \frac{\partial^{2} \bar{u}_{2.0}^{*}}{\partial t^{2}}+\frac{\lambda_{2}}{2\left(\lambda_{1}+\lambda_{2}\right)}=0\end{array}\right.$

The (11) presents the linear correlate to fluid flow pressure to the wall deformation and transverse progressive mechanical wave propagation in space and over time of a disturbance. These are in totally agreement with many numerical models $[7,8]$.

Fluid-structure coupling: The analysis of the fluid-tube coupling, represented on the contact surface, is based on the principle of continuity and adhesion which is given:

$$
\begin{aligned}
& A_{1}=0 ; \quad A_{3}=\frac{A \sqrt{2} I A_{2}}{\sqrt{I}}
\end{aligned}
$$

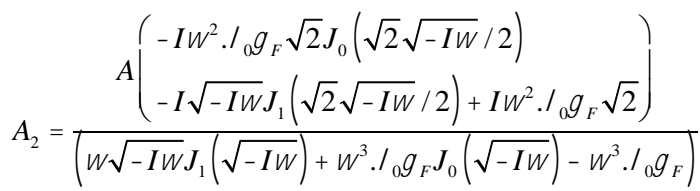

$$
\begin{aligned}
& A=\frac{1}{2} \frac{I\left(\frac{2}{{ }_{1}+{ }_{2}}\right) \sqrt{2}}{\sqrt[3]{I} J_{0}(\sqrt{2} \sqrt{I} / 2)} ; \quad F=\frac{\sqrt{2} \sqrt{I} J_{1}(\sqrt{2} \sqrt{I} / 2)}{{ }^{2} \cdot J_{0}(\sqrt{2} \sqrt{I} / 2)}
\end{aligned}
$$

Here $\left(S_{t} / \quad\right) \quad 0$

\section{Application and interpretations}

In order to investigate the dynamical behaviours of a fluid flow confined in a flexible tube, the geometrical and physical parameters of the simulation are listed in Table1.

Table 1: Parameters of the simulation

\begin{tabular}{|l|c|l|l|}
\hline \multicolumn{1}{|c|}{ Parameter } & \multicolumn{1}{c|}{ Value } & \multicolumn{1}{c|}{ Parameter } & \multicolumn{1}{c|}{ Value } \\
\hline \multicolumn{2}{|c|}{ Fluid (SAE 50W ) } & \multicolumn{2}{c|}{ Tube (PVC) } \\
\hline Density & $\begin{array}{c}902\left[\mathrm{~kg} . \mathrm{m}^{-}\right. \\
3]\end{array}$ & Density & $\begin{array}{c}902\left[\mathrm{~kg} \cdot \mathrm{m}^{-}\right. \\
3]\end{array}$ \\
\hline Dynamic viscosity & $0.86[$ Pa.s] & $\begin{array}{c}\text { Young's } \\
\text { modulus }\end{array}$ & $2[\mathrm{MPa}]$ \\
\hline Inlet velocity & $12\left[{\left.\mathrm{~m} . \mathrm{s}^{-1}\right]}\right.$ & Poisson's ratio & 0.4 \\
\hline Time reference & $31.25 \mathrm{~ms}$ & Length & $0.6[\mathrm{~m}]$ \\
\hline \multicolumn{2}{|c|}{} & Radius (rest) & $7.5[\mathrm{~mm}]$ \\
\cline { 3 - 4 } & & Epaisseur & $2[\mathrm{~mm}]$ \\
\hline
\end{tabular}

It is noted that the parameters listed in Table.1, verify the asymptotic constraints (3): 


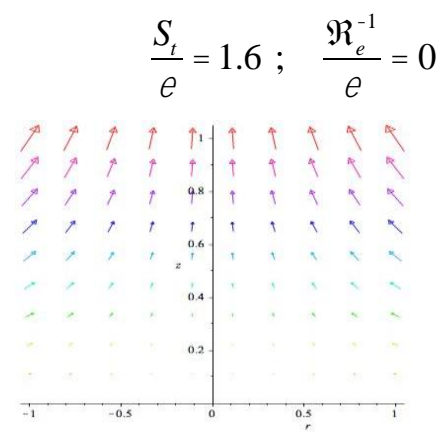

$(a): \quad 8(=6.7)$

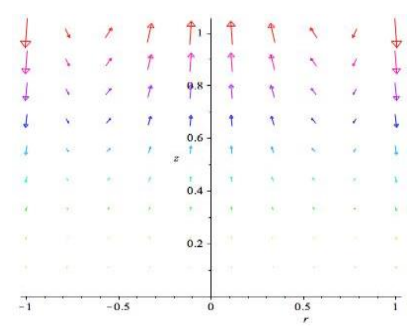

$(c): 8 \square \quad 9.6(=8.5)$

Figure 2. Perturbed fluid velocity field $\left(u^{*}, 0, w^{*}\right)$

Figure 2.a shows that, for the low frequencies, the velocity field keeps the direction of the jet. Exceeding the frequency, the field lines tend to be perpendicular to the direction of the current, which clearly describes the wall tube contraction effects (Figure 2.b). With an increase in frequency, Figure 2.c indicates that the intensity of the inverted field increases and also creates sheared layers, which are represented by Kelvin-Helmholtz instabilities. It should be carry favour the transition to turbulence. This behaviour has been demonstrated in the numerical simulations $[9,10]$. It can be seen that at higher frequency (Figure 3.d), the direction of the perturbed velocity field is reversed, indicating the advent to stop up the fluid flow (aerodynamic pumping).
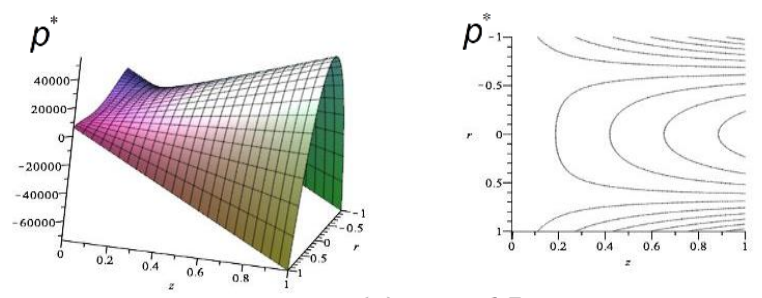

(a) : $\omega=6.7$

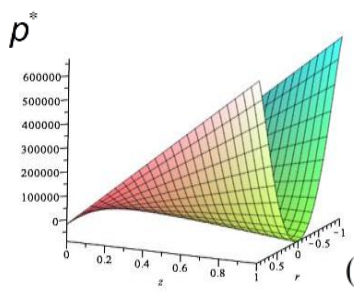

(b) : $\omega=8.7$

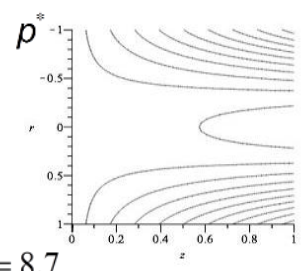

Figure.3. Evolution of the perturbed fluid pressure as a function of $\mathrm{r}$ and $\mathrm{z}$ (left), iso-contours corresponding (right).

Figure 3 illustrates the effect of dimensionless frequency on the axial and radial gradients of the perturbed pressure. We note that the symmetry field is preserved. Also, analysing the gradient pressure along the $\mathrm{z}$-axis reveals a domination of downstream pressure for the higher frequency. Likewise, we find that the radial gradient behaviour highlights the hairpin instability for the flexible tube [11]. Thereby, both gradients exhibit a blockage of the flow for the high frequencies and this confirms the analysis of the velocity field treated in the previous paragraph.

\section{Conclusion}

In this paper, the flow performance of a newtonian fluid confined in a flexible tube is asymptotically investigated. The results allowed us to analyze the effects of the frequencies variation and the wall flexibility over to fluid flow dynamic behavior.

As a result, it has been clearly highlighted that increasing the flow frequency interacting with a flexible wall could generate reverse flow phenomenon.

The opportunities of this work is to take into account the orthoradial displacement of the wall and to analyze an asymmetric behavior of the system.

\section{References}

[1] C. Sainmont, Optimisation d'une aile d'avion a profil adaptable: étude numérique et expérimentale, Thèse, Ecole polytechnique de Montréal, 2009.

[2] O. Prakash and al., Effects of stenosis on nonNewtonian flow of blood in blood vessels, Inter J. of Biomath, 8 (2015), 1550010, 13 pp.

[3] A. Babarit et al, Modélisation numérique et expérimentale d'un système houlomoteur électroactif déformable. $13^{\text {eme }}$ Journees hydrodynamique, France, 2012.

[4] Y. Duanmu and al., Numerical simulations of vortex-induced vibrations of a flexible riser with different aspect ratios in uniform and shear currents. Hydrodynamics, 29 (2017), 1010-1022

[5] W. T. Koiter,: On the foundations of the linear theory of thin elastic shells. Proceedings of the Koni. Nederl. Akade. V. Weten., 73 (1970), 169-195.

[6] S. Laroze, Mécanique Des Structures Tome 1 : Solides Elastiques, Plaques et Coque. Cépaduès, Toulouse, France, 2005

[7] J. Leibinger and al, A path-conservative Osher-type scheme for axially symmetric compressible flows in flexible visco-elastic tubes. Applied Numerical Mathematics, 105 (2016), 47-63.

[8] D. C. Giancoli, Physique générale, volume 3, $2^{\text {eme }}$ édition, De Boeck \& Lacier, Bruxelles, 2004.

[9] M. Lesieur, M and al., Large-eddy simulation of passive scalar diffusion in isotropic turbulence. Physics Fluids , A 1 (1989), 77-95.

[10] L. Peng \& al. 2.5D CFD simulations of gas-solids flow in cylindrical CFB risers. Powder Technology, 291 (2016), 229-243.

[11] P.S. Bernard, The hairpin vortex illusion, Journal of Physics: Conference Series, 318 (2011), 062004. 